\title{
Raman Spectroscopic Studies of Polyfluorenes
}

\author{
Suchi Guha*
}

Department of Physics and Astronomy, University of Missouri-Columbia MO 65211, USA

\begin{abstract}
Polyfluorenes reveal a complex interplay between emissive properties and intra- and intermolecular structure. Vibrational frequencies and intensities determined by Raman spectroscopy are strongly influenced by variations in the backbone as well as side chain conformations. Changes in the structural and electronic properties of two side group substituted polyfluorenes (PF): ethyl-hexyl substituted PF (PF2/6) and dioctyl substituted PF (PF8) as a function of solvent, thermal cycling, and hydrostatic pressure via Raman scattering are presented. The vibrational frequencies and intensities of fluorene oligomers with various alkyl side chain conformations are calculated using hybrid density-functional theory. A comparison of the computed vibrational spectra of single chain fluorene oligomers with our experimental data shows that the conformational isomers in PF8 are a direct consequence of the side chain conformation.
\end{abstract}

\section{INTRODUCTION}

The addition of solubilizing side chains to conjugated polymers has resulted in numerous processable polymers that display a complex interplay between the molecular microstructure and observed photophysics. Poly paraphenylene (PPP) based conjugated molecules and polymers are typically blue emitters. Blue electroluminescent materials are of particular interest for organic displays since blue light can be down-converted into red and green by color-changing media (fluorescent dyes). Polyfluorenes (PFs) represent a unique class of PPP-based blue-emitting polymers with intriguing structure-property relationships. Although PFs have one of the highest luminescence quantum yields, commercialization of these materials in light-emitting diodes and displays is yet to be realized. Molecular level attributes such as local chain structure and side chain conformations in these systems strongly impact transport and device characteristics. In the past decade many research groups have focused on the structural studies of PFs using x-ray scattering [1, 2], smallangle neutron scattering [3], and optical spectroscopy [4-7].

Side chain substitution, in addition to improving the solution processing and film forming characteristics, confers new functionality, and dictates the backbone conformation in PFs. These side chains give rise to a rich array of mesomorphic behavior with the appearance of the nematic liquid crystalline (n- $L C$ ) phase $[8,9]$. In the PF family, poly (9,9-(di n, n-octyl) fluorene) (PF8) and poly (9,9-(di ethyl-hexyl) fluorene) (PF2/6) have received a lot of attention mainly due to their mesomorphism and crystalline phases. PF2/6 in particular has an unusual structure-property relation: despite the molecular-level disorder that is inherent due to two possible enantiomers and isomers, thermally annealed films of PF2/6 exhibit an ordered hexagonal phase with very large coherence lengths [2]. PF2/6 has a limited number of conformational isomers that form a five-fold helix [10]. The optical

*Address correspondence to this author at the Department of Physics \& Astronomy, University of Missouri-Columbia, USA; Tel: 1-573-884-3687; Fax: 1-573-882-4195; E-mail: guhas@missouri.edu properties of PF2/6 are relatively insensitive to the exact crystallographic state and thermal history. In contrast, PF8 shows many crystalline phases that depend upon the thermal history and the nature of the solvent $[11,12]$. PF8 is also known for its presence of a low-energy emitting beta phase, which dominates the optical emission $[5,9]$. Time-resolved photoluminescence studies reveal a fast transfer of excitation to this phase and explain how a small fraction of this phase may dominate the emission [13].

The application of Raman scattering in PFs is particularly useful for resolving ambiguities regarding chain conformations and crystalline phases in PFs. Both the vibrational frequencies and intensities determined by Raman spectroscopy are strongly influenced by variations in the backbone as well as side chain conformations. Specifically in PFs there is evidence of local inhomogeneities in the structural order that gives rise to a variety of conformational isomers with disorder. Recent Raman scattering work (both theoretical and experimental) focusing on the conformations of alkyl side chains in PF8 show that the nature of the polymer backbone in thermally induced crystalline phases is a direct consequence of the side chain conformations $[11,14]$. In addition to structural studies, Raman scattering has also played a major role in determining electron-phonon interactions in PFs as a function of hydrostatic pressure [15], and in the presence of injected and photogenerated charge carriers [16].

This paper is a review of light scattering studies of both PF8 and PF2/6 as a function of thermal cycling and hydrostatic pressure, and is organized as follows; Section II describes our experimental and theoretical methodologies. In Section III we present Raman scattering studies of PF8 and PF2/6, which reveal changes in conformations induced by thermal cycling. In this Section we also compare our theoretical modeling of the vibrational spectra with experimental results focusing on the changes in side-and backbone chain conformation in PF8 upon crystallization. In Section IV we present our hydrostatic pressure dependent studies of PF2/6. This is followed by a summary in Section V. 


\section{METHODOLOGY}

\section{Experimental Procedure}

The PF8 sample was obtained from American Dye Source (BE-129) and dissolved in solvents like p-xylene or toluene and then dried. The starting solutions were prepared with $10 \mathrm{mg}$ of PF8 in $1 \mathrm{ml}$ of the solvent (p-xylene or toluene). Bulk samples were obtained by repeatedly adding solution, as it dried, into small channels. A large thick residue eventually formed and pieces of PF8 were then used for the Raman measurements. The PF2/6 samples were prepared using standard procedures reported previously [17]. PF2/6 may be glassy or semicrystalline at room temperature with a nominal glass transition for the amorphous component at 80 ${ }^{\circ} \mathrm{C}$, a crystalline to nematic- $L C$ phase transition just above $165{ }^{\circ} \mathrm{C}$, and the reverse transition on cooling at $\sim 132{ }^{\circ} \mathrm{C}$. There is considerable time-and temperature-dependent hysteresis on cooling [2].

The Raman spectra were collected by an Invia Renishaw spectrometer attached to a confocal microscope with a $\times 50$ long objective and a $785 \mathrm{~nm}$ line of a diode laser as the excitation wavelength. Typical laser power was a few $\mathrm{mW}$ on the sample. The samples were affixed to a stainless steel sample holder of a Linkam LTS350 microscope hot-cold stage for thermal cycling studies. The pressure studies were conducted in a Merrill-Bassett-type diamond-anvil cell (DAC) with cryogenically loaded argon as the pressure medium. The shift in the luminescence spectrum from a small chip of ruby that was included in the sample chamber was used for determining the pressure. For Raman scattering studies under pressure, a Spex triplemate spectrometer along with the $647.1 \mathrm{~nm}$ line of a $\mathrm{Kr}+$ laser were employed. The pressure studies reported here are only from PF2/6. The Raman studies were measured from a powder sample of PF2/6 in the $\mathrm{DAC}$, whereas the photoluminescence (PL) studies were from both powder and film. The film was prepared by dropcasting PF2/6 dissolved in spectroscopic grade dichloromethane directly onto the surface of the bottom diamond of the DAC. The sticking coefficient of the film on diamond is very poor and thus the film experiences a hydrostatic pressure environment. The low temperature pressure studies were conducted in a closed cycle helium refrigerator.

\section{Computation}

All theoretical computations of fluorene oligomers were performed with hybrid density-functional theory in the GAUSSIAN03 program [18]. We employed the B3LYP (Becke's three parameter hybrid) functional to perform geometry optimization and calculations of force constants, dipole moments, and polarizability derivatives combined with the polarized $3-21 \mathrm{G}^{*}$ basis set [19]. The Raman spectrum calculations are performed in two steps; first the force constant matrix is evaluated and then the eigenvalue equation is solved to obtain the eigenvalues and eigenvectors. Details of our calculations are discussed in Ref. [14].

The Raman frequencies were calculated from fluorene monomers for limiting conformations of the n-octyl and ethyl-hexyl side chain conformation. The vibrational frequencies of a fluorene dimer, trimer, and tetramer with no side chain substitution were calculated by varying the torsional angle between monomers as test cases for various conformational isomers in PF8.

\section{TEMPERATURE DEPENDENCE OF THE RAMAN SPECTRA: PF8 AND PF2/6}

\section{Origin of the Raman Peaks}

Fig. (1) shows the Raman spectrum of as-is bulk PF8 at room temperature (r.t.) in the $100-1600 \mathrm{~cm}^{-1}$ range. The intensities of the various Raman peaks plotted in the three regions are not to scale. The Raman spectrum of PF is characterized by numerous intramolecular $\mathrm{C}-\mathrm{C} / \mathrm{C}-\mathrm{H}$ stretch and bend modes spanning from 100 to $1600 \mathrm{~cm}^{-1}$. The Raman peaks in the $1600 \mathrm{~cm}^{-1}$ region arise from an intraring C-C stretch frequency and is best fit with two peaks: an overwhelmingly dominant peak at $1605 \mathrm{~cm}^{-1}$ and at least one or two weak peaks in the range of $1570-1600 \mathrm{~cm}^{-1}$ [15]. These weaker peaks correspond to a breathing motion of the pentagon within the monomer. The Raman frequencies in the $1250-1350 \mathrm{~cm}^{-1}$ region are associated with the backbone C-C stretch motion; the peak at $1342 \mathrm{~cm}^{-1}$ is the C-C stretch mode from within the monomer unit [14]. The $\mathrm{C}-\mathrm{C}$ stretch mode between adjacent monomers splits into two bands, appearing at 1280 and $1308 \mathrm{~cm}^{-1}$ as shown by the dotted lines in Fig. (1). The $1257 \mathrm{~cm}^{-1}$ corresponds to a $\mathrm{C}-\mathrm{H}$ bend type motion. The weak peak at $1420 \mathrm{~cm}^{-1}$ corresponds to a combined C-H bend and $\mathrm{C}-\mathrm{C}$ stretch between adjacent monomers.

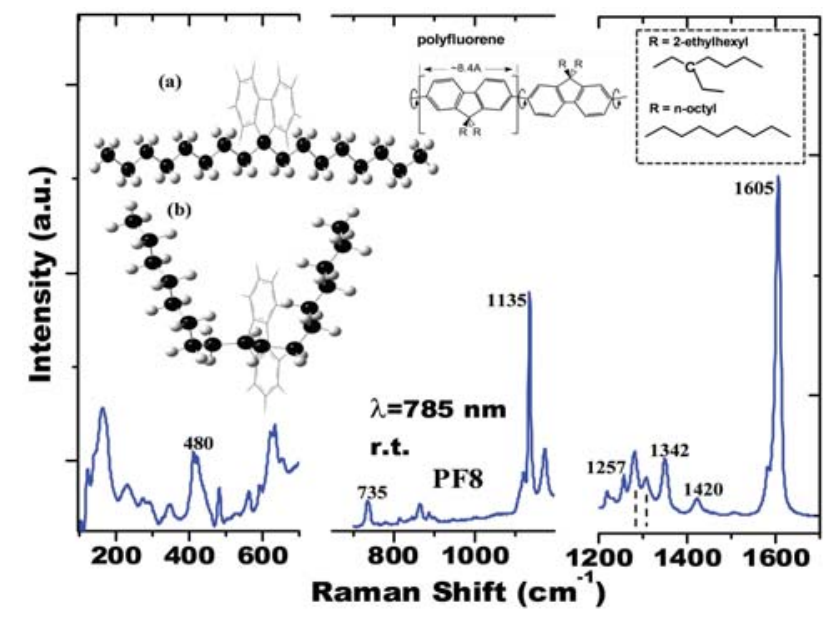

Fig. (1). Raman spectrum of as-is PF8. The inset on the right is a sketch of PF with two different side chains. Insets (a) and (b) show a di-octyl fluorene monomer with aaa and agg side chain conformation.

Frequencies in the $1100-1200 \mathrm{~cm}^{-1}$ region are sensitive to side-group substitution; the 1120 and $1135 \mathrm{~cm}^{-1}$ peaks mainly arise from the $\mathrm{C}-\mathrm{H}$ bending modes (either local or between phenyl units). Crystalline fluorene has a strong peak at $743 \mathrm{~cm}^{-1}$, corresponding to the $747 \mathrm{~cm}^{-1}$ fundamental of a biphenyl with $A_{1}$ symmetry, and thus provides sensitivity of this vibration to the planarity of the ground state [20]. In PFs this frequency is slightly down shifted to $735 \mathrm{~cm}^{-1}$. The stronger Raman peak in the $850 \mathrm{~cm}^{-1}$ region is a combination of ring distortion of the phenyl rings and a $\mathrm{C}-\mathrm{C}$ stretch of the bridging carbon. The weaker Raman peaks around $900 \mathrm{~cm}^{-1}$ relate to $\mathrm{C}-\mathrm{C}$ stretch type motion of the alkyl side chains.

The low frequency Raman peaks in the $100-700 \mathrm{~cm}^{-1}$ range originate from the side chains. This region is thus an extremely sensitive indicator of both side chain composition and ordering. The ring torsion mode at $480 \mathrm{~cm}^{-1}$ is seen in all 
PFs with various side group substitutions, and is almost independent of temperature. Since the Raman peaks around $600 \mathrm{~cm}^{-1}$ and in the $100-400 \mathrm{~cm}^{-1}$ range are mainly from alkyl side chains they have very different signatures for various side chain substituted PFs. To illustrate this point we plot the low frequency Raman spectra of both PF8 and PF2/6 in Fig. (2). The as-is sample refers to the pristine polymer and the thermally-cycled films were measured at r.t., after slowly heating the polymer to its $n-L C$ phase and cooling it back to r.t. The calculated spectra are from fluorene monomers with the appropriate side chain attached to the bridging carbon atom. For PF2/6 only one configuration of the ethylhexyl side chain corresponding to the lowest energy was considered. In PF8, we considered three different types of octyl conformations: all anti (aaa), anti-gauche-gauche (agg), and end gauche (aag); the former two configurations are shown in the inset of Fig. (1).
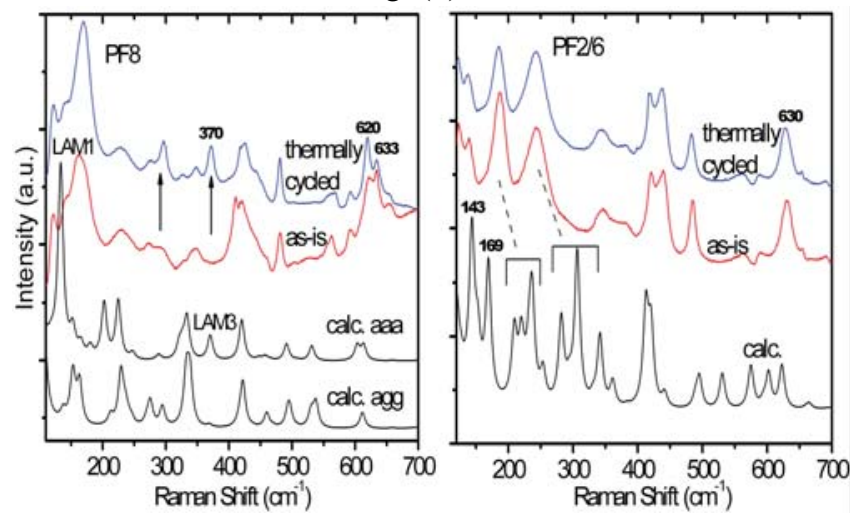

Fig. (2). Left: top two spectra are experimental Raman data of bulk as-is PF8 cast from p-xylene at r.t. and after thermal cycling. The bottom spectra are calculated for two side chain monomer conformations, aaa and agg. Right: experimental Raman data of as-is and thermally cycled PF2/6 (top two); the bottom spectrum is of calculated PF2/6 monomer.

The Raman spectrum in the $100-700 \mathrm{~cm}^{-1}$ region shows significant differences between PF8 and PF2/6. Furthermore, the Raman spectrum of PF2/6 upon thermal cycling hardly changes. In contrast, significant changes are seen in PF8, which derive mainly from changes in the conformation of the alkyl side chain. In perfect transplanar n-alkanes the low frequency Raman spectrum shows intense bands that arise from a longitudinal accordion motion (LAM) of the chain skeleton and overtones [21]. The as-is PF8 polymer has a higher fraction of gauche defects in the octyl side chain. The broad peak centered around $340 \mathrm{~cm}^{-1}$ arises from gauche defects in the as-is polymer and it disappears at temperatures upward of $135^{\circ} \mathrm{C}$. Upon annealing two new peaks appear at 290 and $370 \mathrm{~cm}^{-1}$. The latter corresponds to the LAM3 motion of the alkyl chain, representing a higher fraction of all anti side chain conformation. This peak is clearly seen in the calculated monomer spectrum with aaa conformation. With the presence of gauche defects, agg shown here or aag (not shown here), the peaks corresponding to the LAM motion disappear in our calculations. An enhancement of the LAM1 mode $\left(150 \mathrm{~cm}^{-1}\right)$ upon annealing confirms the presence of a higher fraction of anti side chain confirmations. We will come back to this low-energy region of the Raman spectrum in the next section and show how the conformation of the di- octyl side chains impact the actual skeletal conformation in PF8.

PF8 must be assessed both in terms of its crystalline phase as well as its conformational isomers. Previous studies have identified more than five bulk structural phases (including the $n-L C$ phase) [1] and at least three different conformational isomers $\left(\mathrm{C}_{\alpha}, \mathrm{C}_{\beta}, \mathrm{C}_{\gamma}\right)$ that relate to the torsional angle between adjacent monomers [22]. The $\mathrm{C}_{\beta}$, conventionally referred to as the beta phase, is a more planar form with a torsional angle of $165^{\circ}$ between adjacent monomers. The $\mathrm{C}_{\alpha}$ and the $\mathrm{C}_{\gamma}$ conformations are associated with torsional angles of $135^{\circ}$ and $155^{\circ}$, respectively. Although the beta phase is typically present in very small quantities, it dominates the optical emission, where the main PL peak is red-shifted [5].

There are significant differences in the experimental PF2/6 spectrum and the calculated one of the monomer with the ethyl-hexyl side group (Fig. 2). Many of these may be explained by differences in the (calculated) gas phase spectrum versus the experimental solid state data. The strong 143 $\mathrm{cm}^{-1}$ peak is a torsional motion of the whole molecule and the peak at $169 \mathrm{~cm}^{-1}$ represents an out of plane motion of the phenyl rings. These peaks are much weaker in the solid state and are downshifted in the experimental Stokes Raman spectrum by almost $20 \mathrm{~cm}^{-1}$. The Raman peaks in the $207-240$ $\mathrm{cm}^{-1}$ region originate from a rotation of the $\mathrm{CH}_{3}$ group of the ethyl-hexyl side group. In the experimental spectrum these peaks are not resolved. The peaks in the $300 \mathrm{~cm}^{-1}$ region represent bending of the hexyl group; this motion is restricted in the solid state and thus the experimental values lie at a slightly lower frequency. The calculated Raman peak at 412 $\mathrm{cm}^{-1}$ representing the $\mathrm{C}-\mathrm{C}$ stretch motion of the hexyl chain and the ring torsion motion at $420 \mathrm{~cm}^{-1}$ are barely resolved.

The $630 \mathrm{~cm}^{-1}$ region shows subtle differences in PF8 and PF2/6. There are predominantly two Raman peaks in this region; the low frequency corresponds to a stretching motion of the bridging $\mathrm{C}$ atom connected to the first $\mathrm{CH}_{2}$ group of the alkyl chain and the higher frequency peak involves a torsional motion of the phenyl rings. Shorter alkyl chains such as ethyl-hexyl or an octyl group with two gauche defects in PFs is characterized only by the higher frequency Raman peak. PF2/6 is thus characterized by only one peak at $630 \mathrm{~cm}^{-1}$. The relative ratio of the 620 and $633 \mathrm{~cm}^{-1}$ Raman peaks in PF8 further track the presence of the beta phase [14]. Upon thermal cycling there is a reduction of the beta phase which is seen as an enhancement of the $620 \mathrm{~cm}^{-1}$ peak.

\section{Backbone Conformation}

Structural phase changes as a function of temperature are quite varied in PF2/6 and PF8. X-ray diffraction (XRD) studies of PF $2 / 6$ show that the kinetics to the ordered phase is extremely sluggish. More than $12 \mathrm{~h}$ are required to anneal the polymer from r.t. to its glass transition temperature $\sim 80$ ${ }^{\circ} \mathrm{C}$ for initiating interchain ordering [2]. The structural phase behavior of PF8 as a function of temperature is quite complex; polymorphism in PF8 is strongly dependent upon the solvent used and the thermal history [23]. Mesophase transformation to the $\alpha$ ' polymorph competes with $\alpha$ crystallization on cooling. The $\alpha$ phase is similar to the $\alpha$ polymorph, and in addition it is exceptionally well oriented with respect to the surface normal [1]. 
The Raman spectra of PF8 presented here was cast from p-xylene. Holding and cooling times for temperature dependent Raman studies in PF2/6 and PF8 were similar to the ones used in the XRD studies $[2,23]$. The as-is PF8 appears in a metastable structure at r. t.; PF8 cast from p-xylene is marked by an intermediate M-type phase upon cooling the polymer at r.t. from its $\mathrm{n}-L C$ phase. In terms of the local skeletal structure, PF8 moves from a $\mathrm{C}_{\alpha} / \mathrm{C}_{\beta}$ admixture at the start to $\mathrm{C}_{\gamma}$ and $\mathrm{C}_{\alpha}$ type isomers at the end of the thermal cycle. As pointed out earlier, these various isomers in PF8 are characterized by specific torsional angles between the adjacent units. PF $2 / 6$ in contrast, moves very slowly to an overall ordered hexagonal phase at the end of the thermal cycle.

Fig. (3) shows the Raman spectra of both PF8 and PF2/6 at $50{ }^{\circ} \mathrm{C}$ in the $1100-1700 \mathrm{~cm}^{-1}$ region. The $1308 \mathrm{~cm}^{-1}$ peak (depicted by a dashed line) represents the $\mathrm{C}-\mathrm{C}$ stretch motion between adjacent monomers and thus very sensitive to the torsional angle in PF8. The two insets in Fig. (3) show the peak position of this Raman mode as a function of temperature. The filled triangles are the peak positions upon increasing the temperature while the squares represent the cooling cycle. All Raman peaks in PF2/6 show the same trend as seen for the $1308 \mathrm{~cm}^{-1}$ peak. Between 80 and $150{ }^{\circ} \mathrm{C}$, the peak positions remain almost unchanged, suggesting a gradual onset of the cold crystallization process. The Raman frequency positions typically decrease as a function of increasing temperature due to a decrease in the intermolecular and intramolecular quasielastic force constants. Upon cooling almost a linear increase in the Raman frequency is observed. This feature is very well correlated to the XRD data of PF2/6 [2]: the hexagonal phase upon cooling shows enhanced packing and improved long-range order, which presumably has a shorter intermolecular distance due to a contraction of the ordered phase.

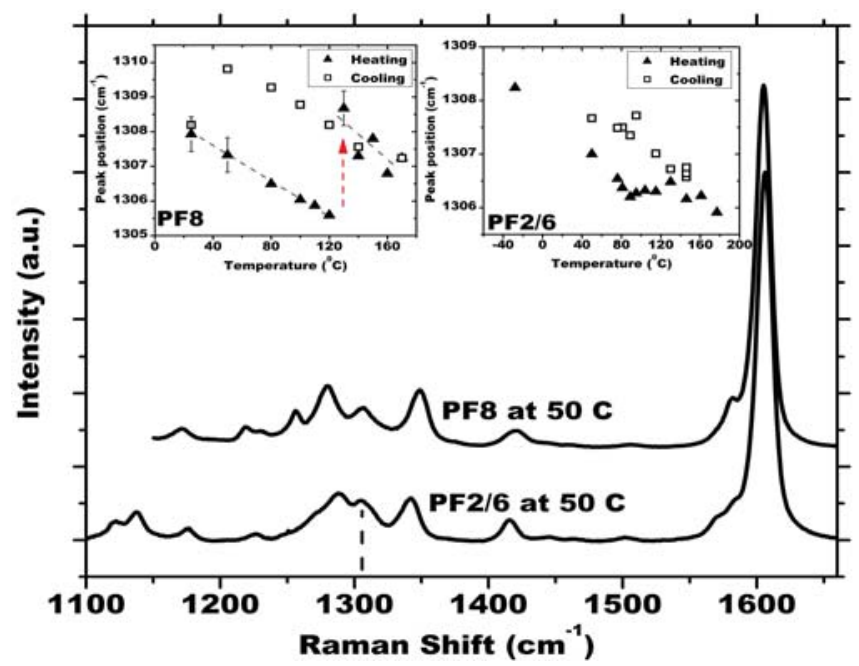

Fig. (3). Raman spectra of PF 8 and $P F 2 / 6$ at $50^{\circ} \mathrm{C}$. The insets show the temperature dependence of the $1308 \mathrm{~cm}^{-1}$ peak both on the heating and cooling cycle.

Along with the various crystalline mesophases being introduced in PF8 upon heating and cooling there are distinct changes in the backbone conformations, which may be tracked by the position of the $1308 \mathrm{~cm}^{-1}$ and the intensity of the $1257 \mathrm{~cm}^{-1}$ Raman peaks. As seen in the inset of Fig. (3), the $1308 \mathrm{~cm}^{-1}$ Raman peak position in PF8 decreases with in- creasing temperatures till $120^{\circ} \mathrm{C}$ and then shows an enhancement by more than $3 \mathrm{~cm}^{-1}$ at $130{ }^{\circ} \mathrm{C}$, marked by the red arrow. This sudden jump reflects a transformation of the $\mathrm{C}_{\beta}$ conformers to the $\mathrm{C}_{\gamma}$ and $\mathrm{C}_{\alpha}$ type conformers. The Raman frequency positions in PF8 as a function of temperature reflect changes in the conformations as well as the crystalline mesophases. The experimental results are consistent with our theoretical calculations. We simulated the Raman spectra of fluorene oligomers (without side chain substitution) by fixing the torsional angle as test cases for the $\mathrm{C}_{\alpha}, \mathrm{C}_{\beta}$, and $\mathrm{C}_{\gamma}$ conformers. Details of these calculations are found in Ref. [14]. The $1308 \mathrm{~cm}^{-1}$ softens by almost $15 \mathrm{~cm}^{-1}$ in the $C_{\beta}$ form compared to the $\mathrm{C}_{\alpha}$ isomer in computed fluorene oligomers. We note that the experimental results show a smaller change in the frequency position of the $1308 \mathrm{~cm}^{-1}$ at $130{ }^{\circ} \mathrm{C}$ compared to our calculations, which may be attributed to a different environment and slightly different torsional angles between our calculations and the experiment. Upon cooling an enhancement of the Raman peak is seen, which is an effect of the overall crystallization. By cooling the sample back to r.t., the $1308 \mathrm{~cm}^{-1}$ Raman peak appears at a slightly higher frequency signaling the presence of $\mathrm{C}_{\gamma}$ chromophores, consistent with our calculations where the $\mathrm{C}_{\gamma}$ chromophore has a slightly higher frequency compared to $\mathrm{C}_{\beta}$.

\section{Alky Side Chain Conformation in PF8}

The presence of the $C_{\beta}$ chromophore is strongly coupled to the overall crystalline phase of the polymer and the conformation of side chains at very short length scales. As seen in Fig. (2), new Raman peaks appear upon a thermal anneal that can be explained on the basis of a specific conformation of the alkyl side chain. Conformation of nalkane chains using vibrational spectroscopy has been heavily studied in the past three decades $[24,25]$. Upon heating nalkanes the crystallization goes through the formation of seeds consisting of weakly bound segments of transplanar chains with short chain segments. In perfect transplanar nalkanes the low frequency Raman spectrum shows intense bands that arise from a LAM motion of the chain skeleton and its overtones. Nonadecane, for example, shows LAM1 and LAM3 modes at 125 and $342 \mathrm{~cm}^{-1}$, respectively [24]. The appearance of the $270 \mathrm{~cm}^{-1}$ and the enhancement of the $150 \mathrm{~cm}^{-1}$ Raman peaks upon thermal cycling in Fig. (2) is an indication of a higher fraction of all anti side chain conformations.

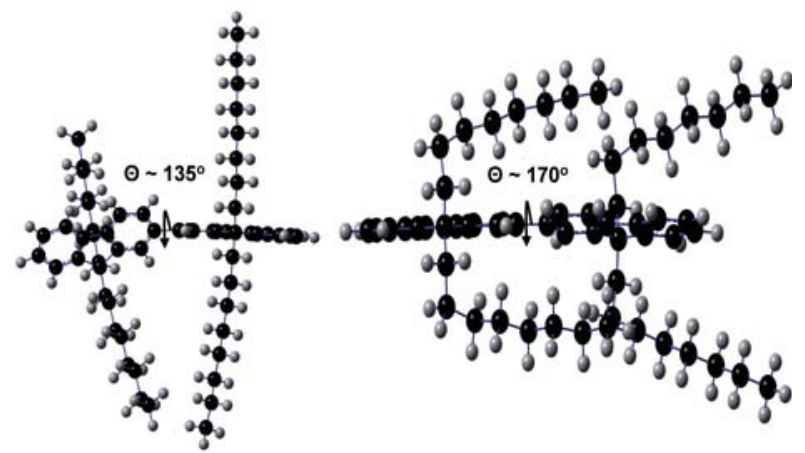

Fig. (4). Fluorene dimer with aaa and agg-type dioctyl side chain.

The next question that arises is the connection between the backbone conformation and the precise side chain conformation. After a thermal anneal the polymer has pre- 
dominantly $\mathrm{C}_{\alpha}$ and $\mathrm{C}_{\gamma}$ type chromophores. At the simplest level the relation between the conformational isomers and the side chain conformation can be understood by looking at the gas phase molecular calculations by Chunwaschirasiri et al. [22]. These calculations show that the $\mathrm{C}_{\beta}$ conformer is stabilized when the alkyl side chains adopt an agg conformation; thus planar backbone units in PF8 require the presence of gauche defects. This is depicted in Fig. (4) for a fluorene dimer with two fixed side chain conformations. With an aaa side chain conformation the dimer adopts a $\mathrm{C}_{\alpha}$ type nonplanar conformation and an agg type side chain conformation leads to a more planar backbone conformation. Although these calculations ignore three-dimensional packing it reveals some basic attributes to the local intrachain structure. To summarize this part: our Raman studies here show that the M-type crystalline phase induced in PF8 upon thermal cycling is compatible with $\mathrm{C}_{\alpha}$ and $\mathrm{C}_{\gamma}$ type chromophores as there is large scale increase in the population of the anti planar conformation of the alkyl side chains. The beta phase in PF8 is incompatible with the overall crystalline phase induced upon thermal cycling.

\section{HYDROSTATIC PRESSURE DEPENDENCE OF THE PL AND RAMAN FREQUENCIES IN PF2/6}

\section{PL Studies Under Pressure}

Hydrostatic pressure allows a study of materials in a region of phase space not accessible by chemical techniques. Application of hydrostatic pressure allows tuning of both intermolecular and intramolecular interactions in polymer chains without any real changes in the chemical make-up [26]. The photophysical studies of conjugated materials under high pressure indicate that enhanced intermolecular interactions produces an increased degree of conjugation [27], red-shift and broadening of the PL [26, 28, 29], destabilization of localized states as in methylated ladder-type PPP [30], and changes in the ring torsion motion as in non-planar para hexaphenyl and other oligophenyls $[31,32]$. In this section we briefly discuss the impact of hydrostatic pressure both on the electronic as well as the vibrational states of PF2/6. Due to the weak Raman signal from PF2/6 under pressure, the Raman studies were carried out from bulk samples; the PL studies under pressure were from both film and bulk samples of PF2/6.

The PL emission of conjugated polymers and molecules typically show a well resolved Franck-Condon (FC) type progression of vibronic sub-bands in addition to the main $\pi$ $\pi^{*}$ electronic transition. Along with the backbone emission, PFs in particular are notorious for a green band emission centered around $2.4 \mathrm{eV}$. The majority of this emission originates at a small number of fluorenone defect (keto type) sites [33]. These defect sites may be incorporated during synthesis or as a result of photo-oxidative degradation process. These sites act as local traps for migrating excitons and strongly reduce the $\mathrm{FC}$ emission. The exact nature of the green emission is still under debate. The work by Dias et al. shows that the intensity and the energy position of the green band emission changes with solvent polarity [34]. They propose the formation of an intramolecular charge transfer complex between PF units and 9-fluorenone to explain the appearance of the new emission band. The green band emission is also seen in single PF chains containing two to four fluorenone units suggesting that these sites are stable emitters even in the absence of any intersegmental interactions; thus the origin of the emission is monomolecular [35].

Fig. (5) shows the PL spectra of PF2/6 (bulk and film) at selected values of pressure during the cycle where the pressure was increased. The individual spectra have been vertically shifted for clarity. Some hysteresis is observed in the PL spectra upon decreasing pressures, details of which are found in Ref. [15]. The vibronic progression seen at 1 bar smears with increasing pressures. The $0-0$ transition is not observed in the 1 bar powder sample due to self absorption effects. A general trend of the PL spectra of conjugated polymers and molecules is a shift to lower energies with increasing pressures. The red shift of the electronic spectra reflects an increase in the degree of conjugation. Presumably under pressure the effective conjugation, which is dependent upon the degree of overlap of the $\pi$-electron wavefunction, is enhanced. The PL virbonic peaks in the film sample shift linearly with pressure with a coefficient of $-2.8 \mathrm{meV} / \mathrm{kbar}$. Although both the powder and film PF2/6 samples red shift under increasing pressure, their signatures are very different. Beyond $18 \mathrm{kbar}$ in the bulk sample the backbone emission is almost absent, and only the $2.4 \mathrm{eV}$ emission originating from keto defects and aggregates dominates the spectrum. The number of keto sites for the 1 bar spectrum (measured under evacuated conditions) and in the DAC would nominally remain the same. However, the keto moieties in the polymer backbone may enhance a twist of the fluorenone units at atmospheric pressures suppressing the migration of excitons to such sites. With enhanced pressures the polymer backbone may be more planarized which allows the migration of energy to the fluorenone defects. Planarization of the backbone under pressure has been observed in other conjugated molecules with a torsional degree of freedom [31].

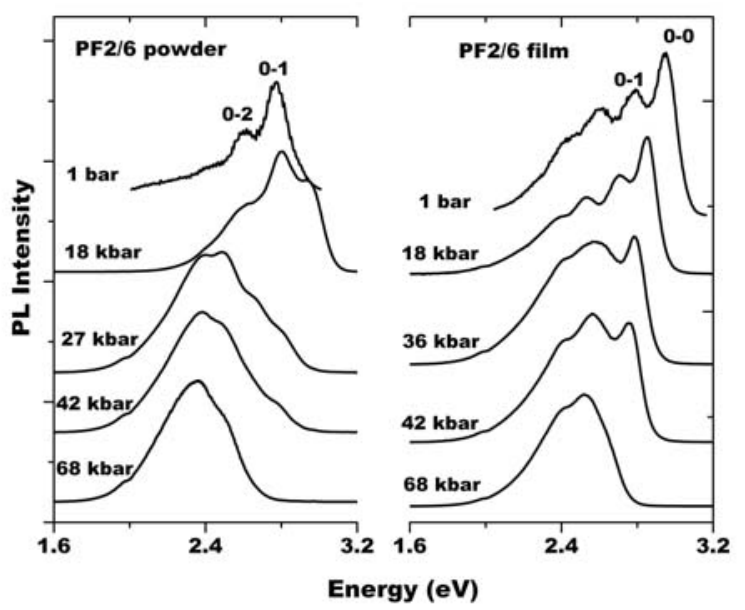

Fig. (5). Photoluminescence spectra of bulk and thin film of PF2/6 measured at r.t. for selected values of hydrostatic pressure.

The difference in the PL spectra at higher pressures between bulk and film samples at the first glance is puzzling. However, one may reconcile these differences by looking at the variations in the morphology of the two samples. The 2.4 $\mathrm{eV}$ emission band in the film appears at pressures excess of $35 \mathrm{kbar}$. The solvent polarity typically impacts the local environment of the polymer chain; a more polar solvent such as 
dicloromethane (used in this work) shows a suppression of the red shifted absorption band [34]. The green band emission in the film and the bulk sample are at slightly different energies signaling the plausibility of more than one origin to this emission. The strong emission in the film beyond 35 kbar is attributed to an aggregate emission with the keto emission showing up as a weak shoulder. The higher aggregation in the film may result from a more compact and ordered morphology. The absence or weak intensity of the keto emission in the film may result from a "pinning" of excitons in ordered regions that prevents diffusion of excitons to the defect states.

\section{Raman Studies Under Pressure}

Fig. (6) shows the Raman spectra of bulk PF2/6 at various pressure values, where the spectra are vertically shifted for clarity. The break in the $x$ axis at $1300 \mathrm{~cm}^{-1}$ denotes the region where the Raman peak from diamond has been removed. The backbone C-C modes are not observable due to the presence of the strong $1330 \mathrm{~cm}^{-1}$ Raman peak from diamonds in the DAC. The inset shows the Raman spectrum at atmospheric pressure and r.t. in the $1000-1700 \mathrm{~cm}^{-1}$ region; below $1500 \mathrm{~cm}^{-1}$ the vertical scale has been expanded by $6 \times$. The description of the displacement pattern of these Raman frequencies was given in Section III.

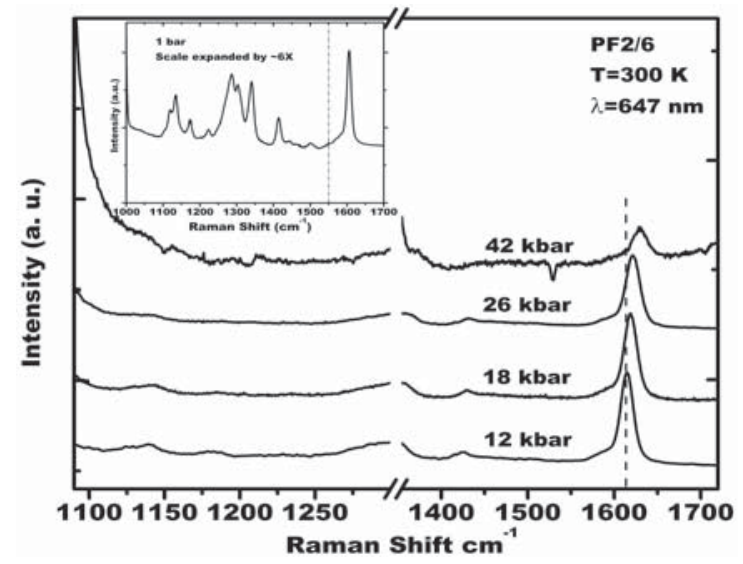

Fig. (6). Raman spectra of PF $2 / 6$ at selected pressures. The inset shows the Raman spectrum at ambient pressure; the vertical scale has been expanded by $6 \times$ below $1500 \mathrm{~cm}^{-1}$.

The Raman frequencies typically harden with increasing pressures. Pressure-induced vibrational frequency shifts in a bulk crystal are often characterized by $\omega_{i}(P) / \omega_{i}(0)=[V(0) / V(P)]^{\gamma_{i}}$, where $\omega_{i}(P)$ is the wavenumber of the $i^{\text {th }}$ mode at the applied pressure $P, V(P)$ is the bulk solid volume at pressure $P$, and $\gamma_{i}$ is the vibrational mode Grüneisen parameter [27]. We note that the Raman scattering of PF2/6 under pressure was measured using the $647 \mathrm{~nm}(1.92 \mathrm{eV})$ of a $\mathrm{Kr}+$ laser. Despite being well below the PL peaks of the backbone and the keto emission, the laser line excites the low energy tail of the PL spectrum (most likely from aggregate emission) due to which the low energy Raman peaks are swamped. Only the $1605 \mathrm{~cm}^{-1}$ can be tracked until higher pressures; the $1100 \mathrm{~cm}^{-1}$ region is observable up to $\sim 20 \mathrm{kbar}$ and the $1417 \mathrm{~cm}^{-1}$ peak is discernable until $\sim 40 \mathrm{kbar}$. By fitting these Raman peaks to a Lorentzian line shape, we find the peak positions to increase linearly with an average pressure coefficient of $+0.5 \mathrm{~cm}^{-1} / \mathrm{kbar}$. Simi- lar pressure coefficients for the vibrational frequencies are seen in other conjugated materials [26].

Along with the increasing Stokes frequency in the Raman spectrum under pressure, the Raman peaks exhibit asymmetric line shapes and an antiresonance effect characteristics of a Breit-Wigner Fano (BWF) resonance. This effect is indicative of a strong electron-phonon interaction between the Raman phonons and the electronic continuum. We have provided a detailed analysis of the BWF phenomenon in PF2/6 under pressure in Ref. [15]. The asymmetry parameter for the $1605 \mathrm{~cm}-1$ increases with pressures beyond 40 kbar. Furthermore, peaks around $1530 \mathrm{~cm}^{-1}$ region appear inverted (slike) as seen in the $42 \mathrm{kbar}$ spectrum (Fig. 6) due to an antiresonance effect. We have cycled the pressure many times to confirm the antiresonance effect; the s-like behavior disappears at lower pressures but appears consistently at higher pressure values.

At 40 kbar both the Raman and PL spectra show significant changes, especially as a function of temperature. Fig. (7) shows the Raman spectrum of PF2/6 at $42 \mathrm{kbar}$ for five different temperatures. The pressure variation in the DAC upon changing the temperature is less than 2 kbar. Fig. (7) inset (a) shows the PL spectra at the same pressure for different temperatures. The relative intensity of the backbone emission decreases with increasing temperature. A significant change occurs between $150 \mathrm{~K}$ and $250 \mathrm{~K}$; the $0-1$ transition beyond $150 \mathrm{~K}$ is completely quenched and the broadband emission at $2.4 \mathrm{eV}$ increases in intensity by more than a factor of three. Along with changes in the PL, the Raman spectrum shows an enhancement of the antiresonance effect between $150 \mathrm{~K}$ and $300 \mathrm{~K}$, where the asymmetry of the 1605 $\mathrm{cm}^{-1}$ peak and the appearance of the inverted s-like peaks in the $1500 \mathrm{~cm}^{-1}$ region are clearly seen. Inset (b) shows the full width at half maximum (FWHM) of the $1605 \mathrm{~cm}^{-1}$ peak as a function of temperature. With increasing temperatures the Raman peaks typically broaden. A bit of a surprise in this data here is an initial decrease and then an enhancement of the peak width beyond 100-150 K. This indicates an overall change in the crystallinity of the polymer.

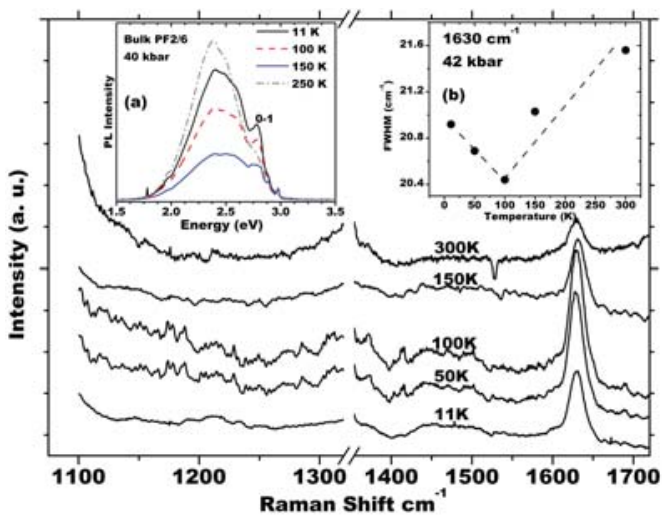

Fig. (7). Raman spectra of PF2/6 at $42 \mathrm{kbar}$ for selected values of temperature. Inset (a) shows the PL spectra at the same pressure for four different temperatures. Inset (b) shows the FWHM of the 1605 $\mathrm{cm}^{-1}$ peak versus temperature.

A recent high pressure XRD work on crystalline fluorene by Heimel et al. [36] indicates a fully reversible phase transition around $36 \mathrm{kbar}$ where the molecular arrangement changes from a herringbone pattern to a $\pi$-stacking geometry. In 
PF2/6 one can conjecture that such a phase transition occurs at $\sim 40 \mathrm{kbar}$ and temperatures beyond $150 \mathrm{~K}$ facilitate an efficient energy transfer to the defect sites, supporting our PL results. Another plausible scenario is that at these pressures the $\mathrm{n}-L C$ phase may be induced at temperatures above 150 $\mathrm{K}$. For obtaining a full insight into the structural changes of PF2/6 at $40 \mathrm{kbar}$, detailed XRD studies are required. Although Raman and PL studies have limitations in extracting the exact structure of materials, our results point in the direction of a phase transition in PF2/6 at pressures beyond 40 kbar. This is also responsible for enhancing the keto emission and the electron-phonon coupling strengths.

\section{SUMMARY}

Raman scattering is a sensitive tool to probe the skeletal conformation governed by inter-fluorene torsional angles and the local side chain structure in PFs. By combining theoretical vibrational spectra calculation of fluorene oligomers and experimental Raman scattering studies of PF8, distinct differences in the alkyl side chain conformation as a function of thermal cycling are revealed. Furthermore, the backbone conformation is intimately tied to the conformation of the alkyl side chain in PF8. It is clearly seen that the beta phase in PF8, which originates from regions of enhanced backbone planarity, requires the presence of gauche defects in the alkyl side chain. Inhomogeneities at these short to intermediate length scales also greatly impact the optical absorption and photoluminescence.

The application of hydrostatic pressure on PF2/6 induces a very efficient energy transfer to fluorenone defect sites beyond $18 \mathrm{kbar}$ in the bulk sample. Upon further increasing the pressures to $40 \mathrm{kbar}$, there is a strong mixing of the Raman modes and the electronic continuum (BWF resonance) that manifests itself as an asymmetric line shape of the 1605 $\mathrm{cm}^{-1}$ Raman peak and an antiresonance effect in the $1500 \mathrm{~cm}^{-}$ ${ }^{1}$ region. Temperature dependence of the PL and Raman spectra at $40 \mathrm{kbar}$ indicates a plausible phase transition. For a quantitative insight into the structural changes of PF2/6 as a function of hydrostatic pressure systematic XRD studies in the future will be invaluable.

\section{ACKNOWLEDGEMENTS}

We thank our collaborators: M. Arif, C. Volz, and C.M. Martin, each of whom played important roles in the development of the field. SG has greatly benefited from fruitful discussions with M. Winokur and U. Scherf. Thanks are also due to M. Chandrasekhar for the use of her research laboratory and useful discussions. We are indebted to U. Scherf and $\mathrm{M}$. Winokur for donation of the PF2/6 sample and the pxylene PF8 samples, respectively. This work was partially supported by the National Science Foundation (ECCS0523656).

\section{REFERENCES}

[1] Chen, S.H; Su, A.C.; Su, C.H; Chen, S.A. Macromolecules, 2005, 38,379 .
[2] Tanto, B.; Guha, S.; Martin, C.M.; Scherf, U.; Winokur, M.J. Macromolecules, 2004, 37, 9438.

[3] Rahman, M.H.; Chen, C-Yu.; Liao, S-Ching; Chen, H-Lung; Tsao, C-Si, Chen, J-Hong; Liao, J-Long; Ivanov, V. A.; Chen, S-An. Macromolecules, 2007, 40, 6572.

[4] Ariu, M.; Lidzey, D.G.; Sims, M.; Cadby, A.J.; Lane, P.A.; Bradley, D.D.C. J. Phys. Condens. Mater., 2002, 14, 9975.

[5] Winokur, M.J.; Slinker, J.; Huber, D.L. Phys. Rev. B, 2003, 67, 184106.

[6] Bernius, M.T.; Inbasekaran, M.; O'Brien, J.; Wu, W. Adv. Mater., 2000, 12, 1737.

[7] Liem, H.; Etchegoin, P.; Whitehead, K.S.; Bradley, D.D.C. J. Appl. Phys., 2002, 92, 1154.

[8] Grell, M.; Bradley, D.D.C.; Ungar, G.; Hill, J.; Whitehead, K.S Macromolecules, 1999, 32, 5810 .

[9] Cadby, A.J.; Lane, P.A.; Mellor, H.; Martin, S.J; Grell, M.; Giebeler, C.; Bradley, D.D.C.; Wohlgenannt, M.; An, C.; Vardeny, Z.V. Phys. Rev. B, 2000, 62, 15604 .

[10] Lieser, G.; Oda, M.; Miteva, T.; Meisel, A.; Nothofer, H.G.; Scherf, U. Macromolecules, 2000, 33, 4490.

[11] Arif, M.; Volz, C.; Guha, S. Phys. Rev. Lett., 2006, 96, 025503.

[12] Chen, S.H.; Chou, H.L.; Su, A.C.; Chen, S. Macromolecules, 2004, 37,6833 .

[13] Khan, A.L.T.; Sreearunothai, P.; Herz, L.M.; Banach, M.J.; Köhler, A. Phys. Rev. B, 2004, 69, 085201

[14] Volz, C.; Arif, M.; Guha, S. J. Chem Phys., 2007, 126, 064905.

[15] Martin, C.M.; Guha, S.; Chandrasekhar, M.; Chandrasekhar, H.R.; Gurntner, R.; Scanduicci de Freitas, P; Scherf, U. Phys. Rev. B, 2003, 68, 115203 .

[16] Arif, M.; Guha, S.; Tsami, A.; Scherf, U. Appl. Phys. Lett., 2007, 90, 252105.

[17] Scherf. U; List, E.J.W. Adv. Mater., 2002, 14, 477.

[18] GAUSSIAN03, Gaussian, Inc., Pittsburgh, PA, 2003.

[19] Becke, A.D. J. Chem. Phys., 1993, 98, 5648.

[20] Negri, F.; Zgierski, M.Z. J. Chem. Phys., 2002, 97, 7124.

[21] Schaufele, R.F.; Shimanouchi, T. J. Chem. Phys., 1967, 47, 3605

[22] Chunwaschirasiri, W.; Tanto, B.; Huber, D.L.; Winokur, M.J. Phys. Rev. Lett., 2005, 94, 107402.

[23] Tanto, B.; Guha, S.; Arif, M.; Winokur, M.J. unpublished.

[24] Zerbi, G,; Magni, R.; Gussoni, M.; Mortiz, K.H.; Bigotto, A.; Dirlikov, S. J. Chem. Phys., 1981, 75, 3175.

[25] Kim, Y.; Strauss, H.L.; Snyder, R.G. J. Phys. Chem., 1989, 93, 7520 .

[26] Guha, S.; Graupner, W.; Resel, R.; Chandrasekhar, M.; Chandrasekhar, H.R.; Glaser, R.; Leising, G. J. Phys. Chem. A, 2001, 105, 6203.

[27] Guha, S.; Graupner, W.; Yang, S.; Chandrasekhar, M.; Chandrasekhar, H.R. in Approaches to Polar Order, Glaser, R. and Kaszynski, P. ACS Symposium Series, 2001, Vol. 798, pp. 127.

[28] Chandrasekhar, M.; Guha, S.; Graupner, W. Adv. Mater., 2001, 13, 613.

[29] Schmidtke, J.P.; Kim, J.S.; Gierschner, J.; Silva, C.; Friend, R.H Phys. Rev. Lett., 2007, 99, 167401.

[30] Yang, S.; Graupner, W.; Guha, S.; Puschnig, P.; Martin, Chandrasekhar, H.R.; Chandrasekhar, M.; Leising, G.; A-Draxl, C. Phys. Rev. Lett. 2000, 85, 2388.

[31] Guha, S.; Graupner, W.; Resel, R.; Chandrasekhar, M.; Chandrasekhar, H.R.; Glaser, R.; Leising, G. Phys. Rev. Lett., 1999, 82, 3625.

[32] Heimel, G.; Somitsch, D; Knoll, P.; Brédas, J.L.; Zojer, E. J. Chem. Phys., 2005, 122, 114544 .

[33] List, E.J.W.; Guentner, R.; de Freitas, P.S.; Scherf, U. Adv. Mater., 2002, 14, 374.

[34] Dias, F.B; Maiti, M; Hintschich, S.I.; Monkman, A.P. J. Chem Phys., 2005, 122, 054904.

[35] Becker, K.; Lupton, J.M.; Feldmann, J.; Nehls, B.S.; Galbrect, F.; Gao, D.; Scherf, U. Adv. Funct. Mater., 2006, 16, 364.

[36] Heimel, G.; Hummer, K.; Draxl, C.A-; Chunwachirasiri, W.; Winokur, M.J.; Hanfland, M.; Oehzelt, M.; Aichholzer, A. Resel, R. Phys. Rev. B, 2006, 73, 024109.

Received: December 14, $2007 \quad$ Revised: June 06, $2008 \quad$ Accepted: June 11, 2008

This is an open access article distributed under the terms of the Creative Commons Attribution License (http://creativecommons.org/licenses/by/2.5/), which permits unrestrictive use, distribution, and reproduction in any medium, provided the original work is properly cited. 